\title{
A Formal Requirements Engineering Framework for CIM Infrastructures Reengineering
}

\author{
Michaël Petit and Eric Dubois \\ Computer Science Department, University of Namur \\ 21 rue Grandgagnage, B-5000 Namur, Belgium, \{mpe,edu\}@info.fundp.ac.be
}

\begin{abstract}
The complexity of the task of designing Computer Integrated Manufacturing (CIM) Systems that can evolve with time is recognized. This complexity definitively makes a methodological approach necessary. The paper proposes a methodology for the task of designing Computer Integrated Manufacturing (CIM) Systems that can evolve with time. It is based on requirements engineering formal languages that enable the expression of (i) the functional goals of the CIM system, (ii) the quality goals on the system (what qualities the system should have), (iii) the specification of alternatives solutions that fulfill the functional goals and (iv) the evaluation of these alternatives w.r.t. the quality goals. To support this methodology we introduce: the Albert II language (for the description of the functional goals) and the $i^{*}$ framework (for the description of the rationales underlying a system design).
\end{abstract}

\section{Keywords}

Functional and Quality Requirements, Business Process Reengineering, Formal Specification Language

\section{INTRODUCTION}

The design of Computer Integrated Manufacturing (CIM) Systems is recognized as a complex task because the complexity of the built systems themselves. This complexity is due a.o. to the large number of components they contain, the diversity of these components (hardware, software or humans) and the complexity of the data involved (e.g. CAD drawings of parts, workschedules, ...) Moreover, CIM systems have to cope with organisational and technological changes that happen during their lifetimes. Methodological approaches are made necessary because of the amount of investment that these systems represent and because errors in the choice and 
implementation of a system may have tremendous financial consequences. This paper proposes a methodology aimed at helping part of the design process and the maintenance of CIM systems.

The process of designing a system is generally decomposed in two main phases: (i) the requirements engineering (RE) activity consists in eliciting and documenting the requirements that users put on the system to be built and its own constituents (devices, software or humans) and (ii) the design engineering (DE) activity consists in the further development and implementation of these constituents. During the RE phase, a number of decisions are made with respect to e.g. the assignment of tasks to humans, devices and software components. These choices are part of a general system design activity (as opposed to the DE activity introduced above).

In this paper, we present research work addressing aspects of the RE activity. The proposed $\mathrm{RE}$ methodology uses a combination of several languages and models in order to help decisions made during the system design process and keep a trace of them for further system maintenance and evolution. We illustrate on a toy CIM case study how the methodology can be used to reengineer an existing system and justify the transition to a slightly modified system. The methodology, applied for the evolution of a system consists in the following steps:

1. Re-modeling of the behaviour of the existing system using a formal specification language.

2. Eliciting and/or re-modeling of the organizational structure and initial rationales for the choices made when first designing the system and formal expression of the functional goals identified in the organizational model in order to verify if they are still currently met in the existing system.

3. Modification of the organizational model to reflect the current situation in terms of goals, their satisfaction and current priorities among them, formalization of the modified functional goals and modeling of alternatives for satisfying the new goals and of the rationales for the choice of the new solution.

4. Formal modeling of the chosen solution (to be implemented).

Phases 1 and 4 of the methodology rely on the use of a formal language called Albert II. The language is briefly described in Section 2 and illustrated with fragments of the specification of a simplified CIM toy system. Phases 2 and 3 are based on the $i^{*}$ framework developed at the University of Toronto. This framework is introduced in Section 3 and illustrated through the handling of the case study. The evolution of goals, some alternatives to the fulfillment of these and the rationales for the choice among them, are presented in Section 4. The final Albert II specification of the new system to be implemented is not presented in this paper due to the lack of space. All along the paper, we use a CIM toy case study to illustrate the proposed methodology. The informal description of this case study follows.

A production cell, under the responsibility of a manager is made of a number of workstations (WS). Each WS can remove a part from its input buffer and transform it into another part which is placed in its output buffer. All buffers can contain no more than one part at any time.

An AGV system is used for the transportation of parts between the WS's. We concentrate on the transportation between 2 WS: WS1 and WS2. Part of the goal of the AGV system is to transport each part made available in the output buffer of WS1 to the input buffer of WS2. The AGV system is composed of a controller and an AGV. The controller has knowledge about the status of the buffers and of the status of the AGV (busy or not). On the basis of this information, it issues orders to the AGV for both removing the part from a WS and delivering it to another WS. 


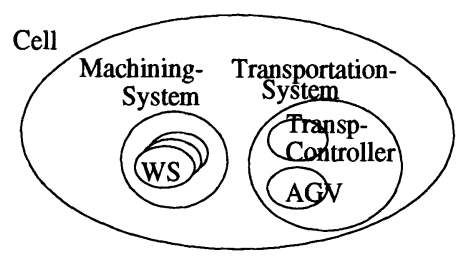

Figure 1 Declaration of the Cell society.

\section{THE Albert II LANGUAGE}

The Albert II specification language is a formal requirements specification language. Its design started around 1992 and the validation of the language constructs has been done through its use in the context of non-trivial systems like Computer Integrated Manufacturing (Dubois et al., 1993) or telecommunication systems (Dubois et al., 1995). Like other formal languages proposed for requirements engineering, Albert II is characterized by its expressiveness and its formality.

- Albert II allows the expression of functional requirements inherent to real-time distributed (cooperative) systems, and

- the semantics of the language relies on some object-oriented variant of real-time temporal logic called Albertmoon (Du Bois, 1995).

The essential feature of Albert II is however its naturalness, i.e. the possibility to map informal statements provided by customers in a straightforward way onto formal Albert II statements. The use of Albert II allows one to avoid the introduction of extra elements in the formal specification which would have no counterpart in customers statements. This means that Albert $\Pi$ is well-suited to the establishment of traceability between informal and formal requirements specifications. It also means that Albert II specifications can be validated by rephrasing them into a natural language text understandable by customers.

In Albert II, functional requirements are expressed in terms of a set of formal statements in typed temporal first order formulas. In order to enhance readability, a specification is organized into units called agents. Logical statements are grouped around agents in order to define the set of admissible behaviors (or lives) they may experience. Logical statements describing an agent are classified into categories, each corresponding to a pattern of property. Such pattern provides guidance in the elicitation and structuring of requirements.

The language is made up of (i) a graphical component for declaring the vocabulary of the application to be considered and (ii) a textual component for constraining the admissible behaviors of agents through logical formulas. For a detailed presentation of Albert II, see (Du Bois, 1995).

\section{Declarations}

The Declarations component consists of a description of the general structure of the composite system in terms of agents as well as of the structure of each individual agent.

A specification consists of a collection of agents. Figure 1 shows the structure of the Cell. Transportation-System is an aggregate of one Transp-Controller and one AGV whereas WS is a population (class) of agents. 


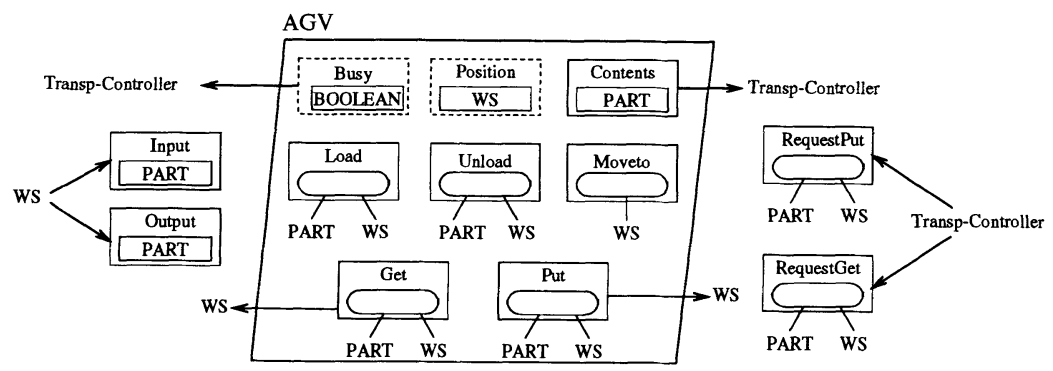

Figure 2 Declaration of the $A G V$ Agent.

The declaration part of an agent consists of the description of its state structure (i.e. the memory of the agent) and the list of actions which may happen during the life of the agent and which may change the state of the agent. State components (graphically depicted with rectangles) are typed and actions (graphically depicted with ovals) can have typed arguments. Types may vary from simple data types to complex data types (recursively built using predefined type constructors).

In the example (see Figure 2), the state of the $A G V$ agent is structured into two individual values (resp. Busy of type BOOLEAN and Position of type WS) and a set of parts (Contents). The AGV may perform five actions: Load, Unload, Moveto, Get and Put. A Get action, e.g. has an argument of type PART which indicates the part transfered on the AGV and an argument of type WS which indicates the identity of the WS where the part is being gotten.

In addition, the graphical notation also expresses visibility relationships linking agents to the outside. Arrows on Figure 2 show how agents make information visible to other agents, e.g., the Busy value of the $A G V$ agent is exported to the Transp-Controller agent; on the contrary, the Position value is shown to no other agents. Arrows also show how agents influence each others' behavior through exportation of actions, e.g., the $A G V$ agent is influenced by the RequestGet actions of the Transp-Controller agent.

\section{Constraints}

Constraints are used for pruning the (usually) infinite set of possible lives associated with the agents of a composite system. The life of an agent is (usually) an infinite alternating sequence of changes (occurrences of actions) and states values. An admissible life will respect:

1. local constraints related to the internal behavior of the agent;

2. cooperation constraints defining how the agent interacts with other agents.

Local constraints are classified under five headings. The use of four of them is illustrated in the example.

Effects of Actions. The effect of an action is expressed through its functional characterization in terms of a mathematical relationship between successive information states (see, e.g. on Figure 3, the effects of the Load and Unload actions)

Action Composition. Composition rules express restrictions on admissible sequences of actions. They also allow to introduce composed actions made of more finer actions. On Figure 3, the illustration of this concept of process is given by the introduction of e.g. a Get action. This 


\section{AGV}

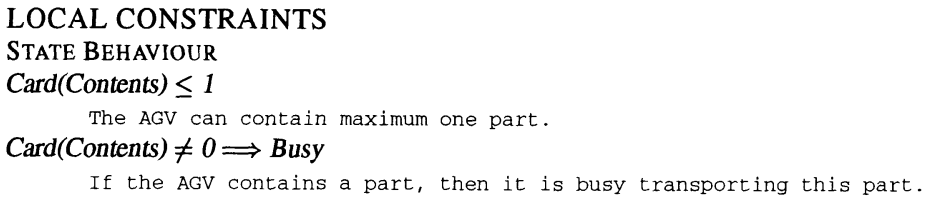

\section{EFFECTS OF ACTIONS}

Load(p,pos): Contents := Add(Contents,p)

The action of loading a part adds this part to the contents of the AGV.

Unload(p,pos): Contents := Remove(Contents,p)

The action of unloading a part removes this part from the contents of the AGV.

Moveto(pos): Position := pos

At the end of a moveto action, the AGV arrives at a particular WS.

Transp-Controller.GetRequest(p,pos): Busy := TRUE

Put(p,pos): Busy := FALSE

The AGV is busy since the moment it receives a get request from the controller until it finishes the corresponding put action.

\section{CAPABILITY}

$\mathcal{F}(\operatorname{Load}(p, p o s) / p o s \neq$ Position $\vee \neg p \in$ pos.Output $) \vee$ Card(Contents) $\neq 0)$ The AGV can only load at a WS where it lies a part which is present in the output buffer of that wS if it does not already contain a part.

$\mathcal{F}(\operatorname{Unload}(p, p o s) / p o s \neq$ Position $\vee \neg p \in$ Contents $\vee \neg$ Card $($ pos.Input $)=0)$ The AGV can only unload a part at a wS where it lies if it does contain the part and if the input buffer of the wS is empty.

$\mathcal{F}(\operatorname{Moveto}(p) /$ Position $=p)$

The AGV can not move to a position that it already occupies.

ACTION COMPOSITION

Get(p,pos) $\leftrightarrow$ Transp-Controller.GetRequest(p,pos); (Moveto(pos) $\oplus$ DAC ); Load(p,pos)

A Get process is initiated by a request of the controller, implies an eventual movement to the specified WS and a load action for the part at that ws.

Put(p,pos) $\leftrightarrow$ Transp-Controller.PutRequest(p,pos); (Moveto(pos) $\oplus$ DAC ) ; Unload(p,pos)

A Put process is initiated by a request of the controller, implies an eventual movement to the specified WS and an unload action for the part at that WS.

Figure 3 Constraints on the AGV agent.

action is made from the occurrence of a GetRequest action (issued by the Transp-Controller) followed by an optional occurrence of Moveto first and by the occurrence of a Load action.

Action Duration. Time constraints can be expressed on the duration of single and composed actions (not illustrated in this example).

Capability Here, we describe ECA (Event-Condition-Action) rules. Besides the circumstances under which an action should or should not occur, the Albert II language also introduces a more non-deterministic characterization where an action is said to be permitted under some circumstances (i.e. may or may not happen). See the three examples on Figure 3.

State Behaviour It is permitted to express properties related to the historical sequence of information states: either static constraints (which are true in all states - usually referred to as 


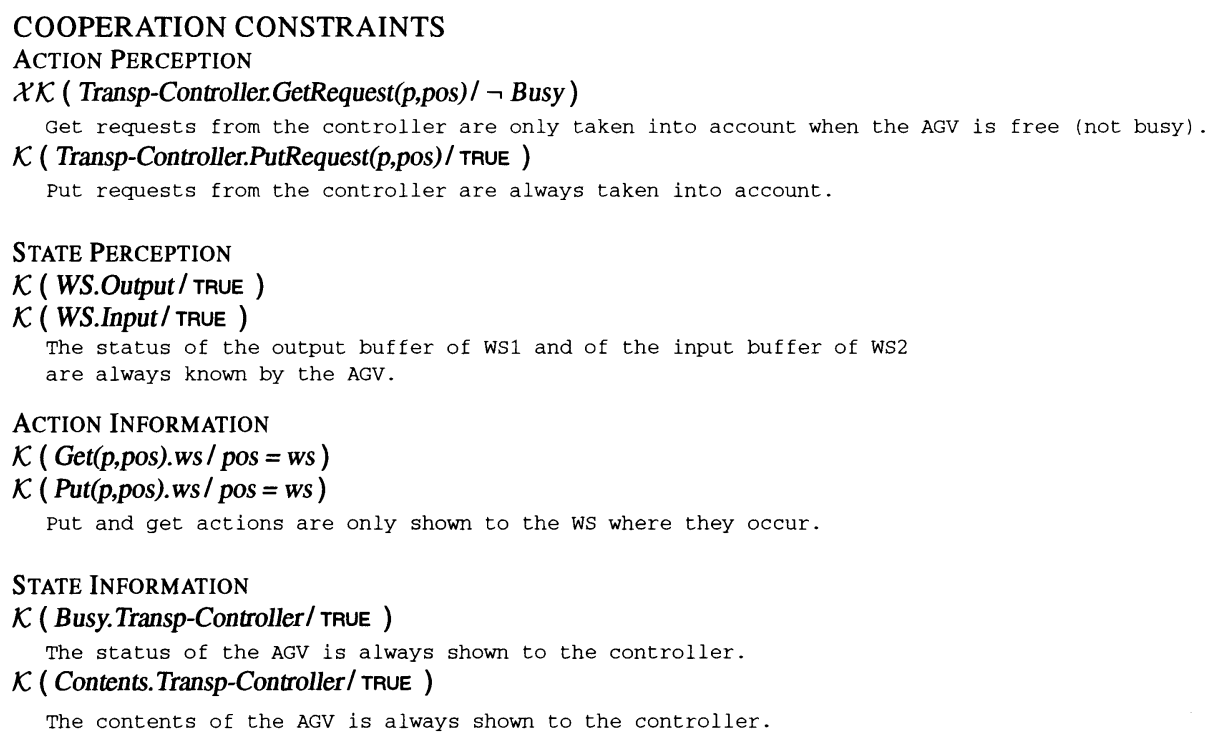

Figure 4 Constraints on the AGV agent (Continued).

invariants) or dynamic constraints (on the evolution of the state). The latter are expressed using temporal connectives. See examples of these constraints on Figure 3.

Cooperation constraints are classified under four headings describing how an agent perceives action performed by other agents (Action Perception), how it can see parts of the state of other agents (State Perception), how it lets other agents know of the actions that it does (Action Information) and how it show parts of its state to other agents (State Information). Perception and information provide the analyst a way to add a dynamic dimension to the importation and exportation relationships between agents expressed in the declaration part of the specification. The headings are illustrated on Figure 4, e.g., the first Action perception constraint defines the conditions under which the $A G V$ agent is influenced by GetRequest actions of the Transp-Controller (in this case, if and only if the AGV is not busy).

\section{ORGANIZATIONAL MODELS: THE $i^{*}$ FRAMEWORK}

In this section, we introduce and illustrate the use of the $i^{*}$ framework for gaining a deeper understanding about the organizational environment, helping to explore alternative patterns of relationships (among software, devices and human components), discovering the implications of these alternatives for each agent, and helping to make tradeoff among the alternatives.

The $i^{*}$ framework provides understanding of the 'why' by modeling organizational relationships that underlie system requirements. Agents are taken to have goals, and use knowhow and 
resources in their attempts to achieve goals. The framework includes two models. In the Strategic Dependency model, agents are modeled as depending on each other for goals to be achieved, tasks to be performed, and resources to be provided. In the Strategic Rationale model, the reasoning that each agent has about its relationships with other agents are described. It supports reasoning about alternative ways for meeting goals, and for evaluating them. Agents are strategic in that they are concerned about opportunities and vulnerabilities.

In the sequel, the $i^{*}$ framework will not be presented in detail. Such presentation can be found in different publications where it is applied in other contexts than CIM (e.g. (Yu and Mylopoulos, 1994) and (Yu et al., 1995)). We will stress on the kind of information conveyed by the framework and suggest how it is complementary to information collected in the Albert II specification.

\subsection{The Strategic Dependency Model}

The Strategic Dependency Model (SDM) supports the description of organizational environments by showing external relationships among actors. On Figure 5, in the context of the case study, such relationships are denoted by labeled links going from one agent to another. As depicted, these agents are those identified in the Albent II specification but, with the exception of the Manager who is an additional agent playing a role at the organisational level. The SDM provides four types of dependency links used to differentiate among the kinds of intentional relationships existing between depender and dependee agents. The use of three of them is illustrated in Figure 5.

In a goal dependency, one agent depends on another to bring about a condition in the world. The depender does not care in what way the dependee accomplishes the condition. In our case study, we have identified a goal dependency called Have parts transferred(WS1,WS2) which expresses that, within the system, it is the hope of the Manager that the Transp-Controller agent will act in such a way so that parts will be transferred from WS1 to WS2. Two other goal dependencies are Begin Transfer by AGV and End Transfer by AGV which indicate that the Transp-Controller depends on the $A G V$ for ensuring the transportation but does not care about how it accomplishes it. Goal dependencies of the $i^{*}$ model are related to some properties associated with the Albert II specification. More precisely, a goal can be associated with theorems that should hold from an Albert II specification (considered in its globality). Those theorems are expressed by introducing global constraints on the action occurrences and state components evolution. For example, the Have parts transferred(WS1,WS2) goal can be formalised as follows:

$(\mathrm{p} \in \mathrm{WS} 1$. Output $\Longrightarrow \diamond \mathrm{p} \in \mathrm{WS} 2$.Input)

Any part $p$ which is in the output of WS1 must be some times latter $(\diamond)$ in the input of ws2.

Since the fulfillment of this goal is depending on the Transp-Controller, we will check its incidences at the level of the Strategic Rationale Model associated with the Transp-Controller (See Section 3.2).

In a resource dependency, the depender depends on the dependee for the availability of an entity (physical or informational). This resource is considered as the finished product of some process followed by the dependee for some other purposes. In the case study, Output is modeled as resource dependency. This means that the Transp-Controller is expected to know about the contents of the output buffer associated with WS1. The existence and the visibility of the output 


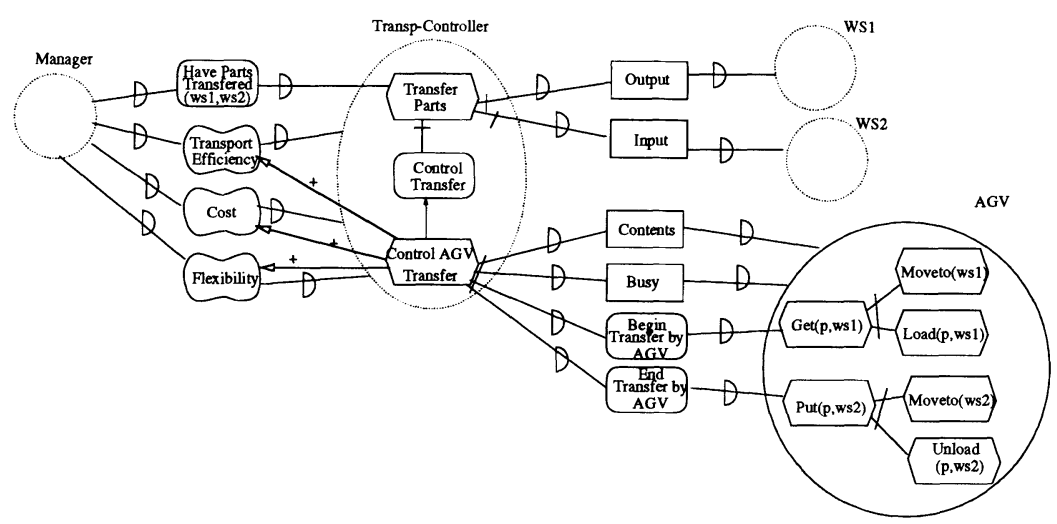

Figure 5 A Strategic Rationale model for the cell example.

buffer is not related to Transp-Controller objective of accessing it but is an intrinsic feature of the WS1. That is why it is related with a resource dependency which allows to trace the information that if the properties of WS1 were changed in the future, the existence or not of the output buffer would influence the behaviour of the Transp-Controller. Again, it is possible to relate the resource goal with some fragments of Albert II. In the specification of WS (not given in the paper), such fragments are:

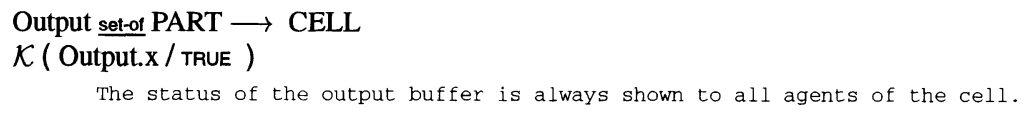

In the case study, other resource dependencies are Input (i.e. the contents of the input buffer associated with WS1), Busy (i.e. the status of the $A G V$ ) and Contents (i.e. the set of parts located on the $A G V$ )

Figure 5 shows also softgoal dependencies: Transport Efficiency, Cost and Flexibility. A softgoal dependency is similar to a goal dependency except that the condition is not sharply defined a priori. For example, the Manager depends on the Transp-Controller for the transport from WS1 to WS2 to be efficient. What is 'efficient' is a matter of interpretation. While the TranspController may do its best to ensure the efficiency of the transport, it is the manager who decides whether it is efficient enough for his purposes. Softgoal dependencies have no direct counterpart in Albert II specifications. However, they heavily influence the design decisions occurring during the elaboration of the solution described by the specification. The nature of these decisions and their impact with respect to softgoal dependencies are further detailed in the next subsection.

Finally, let us mention that there is a fourth kind of link called task dependency in the $i^{*}$ framework. This use of this link, not illustrated in our case study, allows to indicate that the depender depends on the dependee to perform an activity. A task dependency specifies how the task is to be performed, but not why. 


\subsection{The Strategic Rationale Model}

Whereas the Strategic Dependency model gives an external view of how agents depend on each other, the Strategic Rationale Model (SRM) gives a more detailed description of the rationales behind the dependencies. One can answer 'why' questions more precisely. Like in the SDM, intentional elements (goal, resource, task, softgoal) appear in the SRM but are complemented with specific links associated with means-ends relationships and task-decompositions.

In the SRM associated with the Transp-Controller agent depicted in Figure 5, the external goal to Have Parts Transferred(WS1,WS2). is met by the Transfer Parts task which indicates that a certain procedure is followed by the Transp-Controller to this end. This task consists of (represented via task-decomposition links): controlling the transfer, accessing to the content of the output buffer of WS1 and to the content of the input buffer of WS1. Control Transfer appears as a goal to be met. This means that freedom is left to the designer of the system about choosing the way to meet it. In our case, it has been decided that this goal can be met (represented via a means-ends link) by controlling an AGV in charge of the transfer. This Control AGV Transfer task is again decomposed in two goals and two resources dependencies.

As we mentioned, several alternatives could have been imagined for meeting the Control Transfer goal. The evaluation of the different possibilities as well as the selection of a specific one have to be related to the identified softgoals. In the case study, we have already noticed that the manager wants a transportation system being efficient, cost effective and flexible. Different ways of transferring parts (i.e., different organizational configurations) may be evaluated as contributing positively or negatively to these goals. In our case, using an AGV system is considered to be good (or at least sufficient) for the efficiency (tested by the means of simulations). The flexibility* for the AGV is also considered to be good because of its ability to link easily any two workstations in both directions. Cost-effectiveness is also good since only one transporter is needed to fulfill the transportation requirements between all WS of the cell, so the hardware costs were limited and compensated the higher investment necessary for the AGV controller.

Like for the SDM, elements of the SRM model can be linked to fragments of the Albert II specification. With respect to the formal specification of the Transp-Controller given in the annex, the following links can be established:

- With the Transfer-Parts task are associated fragments of Albert II specification related to the perception of the content of the input and output workstations buffers and the identification of the Transport action (see statements 1 and 10).

- With the Control Transfer goal are associated specifications related to the conditions under which the transfer can occur (see statements 4-7).

- With the Control AGV Transfer task are associated specifications associated with the decomposition of the Transport action in terms of finer actions controlling the AGV (see statements 2, 3, 7, 9 and 11)

Finally, on Figure 5, one can also see the SRM associated with the $A G V$. Typically, at its level, only tasks are identified since AGV's have 'hard-coded' behaviours which do not offer flexibility in their design (viz no intermediate goals can be identified).

\footnotetext{
*When evaluating flexibility, different facets of it can be envisaged. We consider here only one of them: the number of possible ways to perform the same tasks (in our case, alternative routings of parts between WS that produce the same end-results).
} 


\section{CHANGES MANAGEMENT AND CONCLUSIONS}

Basically, if we are spending time and effort to capture all the requirements (both the 'whats' and the 'whys') underlying a system, this is because we are convinced that all this information will be of a great interest when the system will have to evolve and be maintained in the future. If we aim at building real flexible systems, we need to offer facilities for changes management, in particular at the requirements engineering level.

Hereafter, we examine how the case-study system could evolve in response to a change in the load of the cell (functional aspects) and in the priority among soft goals (non-functional aspects). We also indicate which information is required for managing this change.

Let us suppose that a change in the load of the cell has increased the transport requirements (in quantity) between WS1 and WS2. As discussed in Section 3, the current AGV system was initially considered as satisfactory with respect to efficiency, flexibility and cost-effectiveness. But now, the efficiency of the transport system is too unsatisfactory since most of the time, the part produced by WS1 has to wait in the buffer before to be transported to WS2. This waiting time causes delay to WS1 because no other part can be produced while the buffer is occupied. Several alternative solutions to this problem have been imagined. Only one of them is described here.

A solution where a conveyor is used for only the transportation of parts from WS1 and WS2 and where the AGV remaining responsible for the rest of the transports in the cell was imagined. The Albert II specification is not given in this paper. A new $i^{*}$ SR model is elaborated to compare both alternatives and contains the following information.

The conveyor solution is certainly worse than the AGV w.r.t. cost since the AGV system is retained (and slightly modified) and an extra investment has to be engaged for the implementation of the conveyor. The flexibility of the cell (as defined above) is worsened since with the AGV system, though this possibility was not used, parts could be easily transported from any WS to any other WS. With the conveyor system, there exists a fixed link from WS1 to WS2 limiting the flexibility of transport. The efficiency is far better for several reasons: parts in WS1 do not have to wait for the AGV to come and load them anymore, the AGV now has to handle less transport requests than before and the efficiency of WS1 is better because the conveyor acts as a buffer between WS1 and WS2. This important increase in efficiency compensates for the drawbacks of this solution. This reflects a change in the priority among soft-goals.

The last two steps of our methodology consist in the elaboration of the specification of the new system to be implemented (not given in this paper) and its implementation. However, as suggested in (Yu et al., 1995), the elaboration of this specification for an alternative solution may help to refine the $i^{*}$ models, so that the elaboration of the Albert II specifications for several alternatives could help in the refinement of the $i^{*}$ models containing the rationales for the choice of the new solution.

In order to make this approach effective, we are planning to develop supporting tools allowing the editing of Albert II and $i^{*}$ models together with their storage in a repository (called ConceptBase (Jarke et al., 1995)) where are maintained the traceability links (sketched in Section 3.2) existing between the two descriptions. In another research project, besides the Albert II editor (already available on Windows platforms), other specialised tools are developed for supporting the verification and validation of formal Albert II specifications.

In future work, we would like to extend the presented framework in such a way that the reuse of generic CIM components is encouraged. To this end, we plan to develop a library of com- 
ponents (specified in Albert II) accompanied by information (expressed in $i^{*}$ ) related to the rationales underlying their design.

\section{Acknowledgment}

We are grateful to Eric Yu for his comments on the $i^{*}$ model and the fruitful cooperation established with him within the framework of the European/Canadian ISC-CAN-080 project on Cooperative Information Systems. European Community also supported this research under Project 8319 (Modelage).

\section{REFERENCES}

Du Bois, Philippe (1995) The Albert II Language: On the Design and the Use of a Formal Specification Language for Requirements Analysis. PhD thesis, Computer Science Department, University of Namur, Namur (Belgique), September 1995.

Chung, Lawrence (1993) Representing and using Non-functional Requirements: a ProcessOriented Approach. PhD thesis, Computer Science Department, University of Toronto, Toronto (Canada), June 1993.

Dubois, Eric and Du Bois, Philippe and Petit, Michaël (1993) Elicitating and formalising requirements for CIM information systems. In C. Rolland, F. Bodart, and C. Cauvet, editors, Proc. of the 5th conference on advanced information systems engineering - CAiSE'93, pages 252-274, Paris (France), June 8-11, 1993. LNCS 685, Springer-Verlag.

Dubois, Eric and Du Bois, Philippe and Zeippen, Jean-Marc (1995) A formal requirements engineering method for real-time, concurrent, and distributed systems. In Proc. of ICSE-17 Workshop on Formal Methods Applications in Software Engineering, Seattle WA, April 24$25,1995$.

Greenspan, Sol J. and Borgida, Alexander and Mylopoulos, John (1986) A requirements modeling language. Information Systems, 11(1):9-23.

Jarke, M. and Gallersdörfer, R. and Jeusfeld, M.A. and Staudt M. and Eherer S. (1995) ConceptBase - a deductive object base for meta data management. Journal of Intelligent Information Systems, Special Issue on Advances in Deductive Object-Oriented Databases, 4(2):167-192.

Yu, Eric S. K. and Mylopoulos, John (1994) From E-R to "A-R" - modelling strategic actor relationships for business process reengineering. In Proc. of the 13th International Conference on the Entity-Relationship Approach - ER'94, Manchester (UK), December 13-16, 1994.

Yu, Eric and Du Bois, Philippe and Dubois, Eric and John Mylopoulos (1995) From organization models to system requirements - a "cooperating agents" approach. In Proc. of the Third International Conference on Cooperative Information Systems - CoopIS-95, Vienna (Austria), May 9-12, 1995. University of Toronto Press inc.

\section{APPENDIX 1 Albent II SPECIFICATION OF THE CELL SYSTEM}

Transportation problem between 2 workstations. For the specification of the AGV agent, see section 2 . The specification of the ws agent is omited due to the lack of place.

\section{Transp-Controller}

DECLARATIONS

STATE COMPONENTS 
WS1 instance-of WS

WS2 instance-of WS

Two particular wS among all the ws of the cell.

Busy instance-ol BOOLEAN

Contents set-of $P A R T \longleftarrow A G V$

Output set-ot PART «WS

Input set-of PART «WS

The controller imports the status (busy or not) and contents of the AGV and the statuses of the input and output buffers of every WS.

ACTIONS

Transport(PART,WS,WS)

(1) The action of transporting a part from a wS to another.

GetRequest(PART,WS) $\rightarrow A G V$

PutRequest(PART,WS) $\longrightarrow A G V$

(2) Requests to the AGV to perform respectively a get and put operation

at a particular wS, for a particular part.

\section{LOCAL CONSTRAINTS}

EFFECTS OF ACTIONS

OperateAGV: Operating := TRUE

\section{CAPABILITY}

$\mathcal{F}$ ( Transport(p,pos1,pos2)/ AGV.Busy)

(3) No transport can occur if the AGV is already busy.

$\mathcal{F}($ Transport $($ p,pos1,pos2 $)$ with pos $1=W S 1 / \neg$ pos2 $=W S 2)$

(4) A transport originating from WS1 can only be destinated to WS2.

$\mathcal{F}($ Transport(p,pos1,pos2) with pos $2=W S 2 / \neg \operatorname{pos} 1=W S 1)$

(5) A transport destinated to wS2 can only originate from WS1.

$\mathcal{F}$ (Transport(p,pos1,pos2)/ $\neg p \in$ pos1.Output)

(6) The transport of a part from a ws to another can not occur if the part

is not present in the output buffer of the origin wS.

$\mathcal{F}$ ( PutRequest(p,pos2)/ $\neg$ Card(pos2.Input) $=0 \vee \neg p \in A G V$.Contents )

(7) The controller can not ask the AGV to put a part in the input of a WS if the input is not empty or if the part is not on the AGV.

\section{ACTION Composition}

Transport(p,pos1,pos2) $\leftrightarrow$ GetRequest(p,pos1); PutRequest(p,pos2)

(8) Performing a transport consists in asking the AGV to get the part

at the origin and put it at the destination.

\section{COOPERATION CONSTRAINTS}

\section{STATE PERCEPTION}

$\mathcal{K}($ AGV.BuSY/TRUE )

$\mathcal{K}($ AGV.Contents / TRUE )

(9) The status and contents of the AGV are always percieved by the controller.

$\mathcal{K}$ (WS1.Output/ TRUE )

$\mathcal{K}$ (WS2.Input/TRUE )

(10) The contents of the output buffer WS1 and input buffer of WS2 are

always percieved by the controller.

\section{ACTION INFORMATION}

$\mathcal{K}($ AGV.GetRequest(p,pos)/TRUE )

$\mathcal{K}$ ( AGV.PutRequest(p,pos)/ TRUE )

(11) Get and put requests are always shown to the AGV. 